\title{
Observation of Weakened V-V Dimers in the Monoclinic Metallic Phase of Strained $\mathrm{VO}_{2}$
}

\author{
J. Laverock, ${ }^{1,2}$ V. Jovic, ${ }^{3}$ A. A. Zakharov, ${ }^{4}$ Y. R. Niu, ${ }^{4}$ S. Kittiwatanakul, ${ }^{5,6}$ B. Westhenry, ${ }^{1}$ \\ J. W. Lu, ${ }^{5}$ S. A. Wolf, ${ }^{5,7}$ and K. E. Smith ${ }^{2,3}$ \\ ${ }^{1}$ H. H. Wills Physics Laboratory, University of Bristol, Tyndall Avenue, Bristol BS8 1TL, United Kingdom \\ ${ }^{2}$ Department of Physics, Boston University, 590 Commonwealth Avenue, Boston, Massachusetts 02215, USA \\ ${ }^{3}$ School of Chemical Sciences and MacDiarmid Institute for Advanced Materials and Nanotechnology, \\ University of Auckland, Auckland 1142, New Zealand \\ ${ }^{4}$ MAX-lab, Lund University, SE-221 00 Lund, Sweden \\ ${ }^{5}$ Department of Materials Science and Engineering, University of Virginia, \\ Charlottesville, Virginia 22904, USA \\ ${ }^{6}$ Department of Physics, Faculty of Science, Chulalongkorn University, Bangkok 10330, Thailand \\ ${ }^{7}$ Department of Physics, University of Virginia, Charlottesville, Virginia 22904, USA
}

(Received 30 July 2018; revised manuscript received 4 October 2018; published 20 December 2018)

\begin{abstract}
Emergent order at mesoscopic length scales in condensed matter can provide fundamental insight into the underlying competing interactions and their relationship with the order parameter. Using spectromicroscopy, we show that mesoscopic stripe order near the metal-insulator transition (MIT) of strained $\mathrm{VO}_{2}$ represents periodic modulations in both crystal symmetry and $\mathrm{V}-\mathrm{V}$ dimerization. Above the MIT, we unexpectedly find the long-range order of $\mathrm{V}-\mathrm{V}$ dimer strength and crystal symmetry become dissociated beyond $\approx 200 \mathrm{~nm}$, whereas the conductivity transition proceeds homogeneously in a narrow temperature range.
\end{abstract}

DOI: 10.1103/PhysRevLett.121.256403

The recent observations of the separation of the electronic and structural transitions of $\mathrm{VO}_{2}$ have shed new light on the microscopic mechanism of the metal-insulator transition (MIT) of this exemplar MIT material [1-3]. Bulk $\mathrm{VO}_{2}$ at low temperature exists in a strongly dimerized (between neighboring $\mathrm{V}$ ions) and insulating monoclinic structure (the $M_{1}$ phase), which transitions to the high symmetry tetragonal rutile $(R)$ phase at $65^{\circ} \mathrm{C}$ concomitant with a huge change in the conductivity of 4 orders of magnitude $[4,5]$. The advantageous properties of this transition [6-8] have placed $\mathrm{VO}_{2}$ at the forefront of exploitable new technologies [6,9], particularly when married with recent advances in its preparation and growth $[10,11]$. However, the coincidence of these large structural and electronic transitions precludes the unambiguous untangling of the mechanism behind the transition, which has led to decades of debate as to whether the MIT is driven by structural instabilities towards dimerization or as a consequence of the effects of strong electron correlation $[5,12,13]$. In this context, the details of the low symmetry monocliniclike metallic phase(s) that develop intermediately between $M_{1}$ and $R$ offer a unique and crucial insight into these questions.

Published by the American Physical Society under the terms of the Creative Commons Attribution 4.0 International license. Further distribution of this work must maintain attribution to the author(s) and the published article's title, journal citation, and DOI.
In addition to the well-known $M_{1}$ and $R$ phases, $\mathrm{VO}_{2}$ is host to a rich set of structural phases in close proximity to its solid-state triple point [8], which are accessible by alloying and/or strain $[10,14,15]$ and are suggestive of a highly contoured energy landscape with competing structural instabilities $[8,16,17]$. For example, whereas each $\mathrm{V}$ ion is paired via dimerization and twisted about its dimer axis in the $M_{1}$ structure [5], the related $M_{2}$ and triclinic $T$ structures both contain alternating strongly and weakly dimerized $\mathrm{V}$ bonds [14]. The similar magnitude of the band gaps in all three of these insulating structures, despite differing $\mathrm{V}-\mathrm{V}$ bonding patterns, has been taken as evidence that electron correlations dominate the MIT [18]. Other apparently distinct phases have also been proposed either as intermediate in the MIT or in close proximity, such as monoclinic metallic $[1,2,19]$ and insulating rutile phases [19-21]. The key to understanding the origin of the MIT in $\mathrm{VO}_{2}$ may lie with a microscopic understanding of such phases, particularly whether $\mathrm{V}-\mathrm{V}$ dimers remain strongly paired despite remaining metallic; recent measurements suggest that the conductivity is intimately linked to the "twisting angle" of the $\mathrm{V}-\mathrm{V}$ pairs [22].

Here, we employ spatially resolved x-ray absorption spectroscopy (XAS) by imaging the secondary electron yield using photoemission electron microscopy (XPEEM) in concert as a spectromicroscopic technique (XASPEEM), which we apply in detail to the intermediate region of the MIT. XAS at the $\mathrm{O} K$ edge in $\mathrm{VO}_{2}$ is sensitive to both $\mathrm{V}-\mathrm{V}$ dimerization through the so-called $d_{\|}$peak, which arises 
due to the absorption of $\mathrm{x}$ rays into unoccupied dimer $\left(a_{1 g}\right)$ orbitals, and to the metallicity of $\mathrm{VO}_{2}$ via the leading edge of the spectrum, which exhibits $\sim 0.2 \mathrm{eV}$ shift between insulating and metallic phases $[23,24]$.

High quality, $300 \mathrm{~nm}$ thin films of $\mathrm{VO}_{2}$ were grown on (110)-oriented rutile $\mathrm{TiO}_{2}$ substrates, leading to biaxial in-plane strain (tensile along $c_{R}$ and compressive along $[110]_{R}$ ) [25-27]. Spectromicroscopic measurements were performed using low energy electron microscopy (LEEM, 7 eV electron energy) [28] and XASPEEM (1.7 eV) [25,29] at the SPELEEM endstation of Beamline I311, MAX-lab (Lund, Sweden), with x-ray energy and position resolutions of $0.2 \mathrm{eV}$ and $40 \mathrm{~nm}$, respectively [25], and diffraction patterns were regularly monitored for electron or X-ray damage [30]. Depth sensitivities of LEEM and XASPEEM are 1 and $5 \mathrm{~nm}$, respectively. Crucially, our biaxially strained $\mathrm{VO}_{2}(110)$ thin films serve two purposes, allowing both the intermediate region of the phase diagram to be explored, and the different coexisting phases to be probed independently but at the same time.

Figure 1 summarizes the main experimental results: LEEM and XASPEEM images are shown at three important stages in the MIT of $\mathrm{VO}_{2}(110)$ [Figs. 1(a)-1(i)], alongside spatially averaged $\mathrm{O} K$ edge XAS [Fig. 1(j)]. The three temperatures chosen represent [1] (i) $51{ }^{\circ} \mathrm{C}$ : insulating, emerging rutilelike stripes; (ii) $58^{\circ} \mathrm{C}$ : stripes fully established, both metallic and insulating monocliniclike phases coexist; and (iii) $69^{\circ} \mathrm{C}$ : fully metallic, small stripes of monocliniclike symmetry persist.

Below the MIT at $24^{\circ} \mathrm{C}$, the characteristic $M_{1}$ phase spectrum is observed in Fig. 1(j), consisting of a peak at $530.0 \mathrm{eV}$ due to $\pi^{*}$ states, followed by a prominent $d_{\|}$peak at $530.7 \mathrm{eV}$ due to $\mathrm{V}-\mathrm{V}$ dimerization [23,24]. The complete suppression of the $d_{\|}$peak above the MIT $\left(97{ }^{\circ} \mathrm{C}\right)$ is due to the absence of $\mathrm{V}-\mathrm{V}$ dimers in the $R$ phase, and the shift downwards by $0.2 \mathrm{eV}$ of the leading edge arises due to its metallicity. At intermediate temperatures, some excess intensity exists at the $d_{\|}$peak, suggestive of the presence of $\mathrm{V}-\mathrm{V}$ dimers. Spectra recorded at temperatures $\geq 57^{\circ} \mathrm{C}$ show the same leading edge and $\pi^{*}$ spectra as rutile $\mathrm{VO}_{2}$, indicating that the sample predominantly contains metallic $\mathrm{VO}_{2}$, despite the persistence of rutile and monoclinic stripes up to $87^{\circ} \mathrm{C}$. However, at intermediate temperatures of $51^{\circ} \mathrm{C}$ and below, the absorption onset follows the insulating monoclinic shape, and the $\pi^{*}$ feature appears to be intermediate between the $M_{1}$ and $R$ phase spectra. These results are in good agreement with our previous LEEM and photoemission measurements on the same $\mathrm{VO}_{2}(110)$ system, where fully metallic photoemission spectra were observed at $61{ }^{\circ} \mathrm{C}$ and above [1].

Structural stripes oriented along the $c_{R}$ axis are clearly observed in the LEEM images, Figs. 1(a), 1(d), and 1(g). The contrast in the LEEM is due to diffraction contrast, in which bright intensity corresponds to the rutilelike phase [1]. Corresponding contrast is visible in the XASPEEM
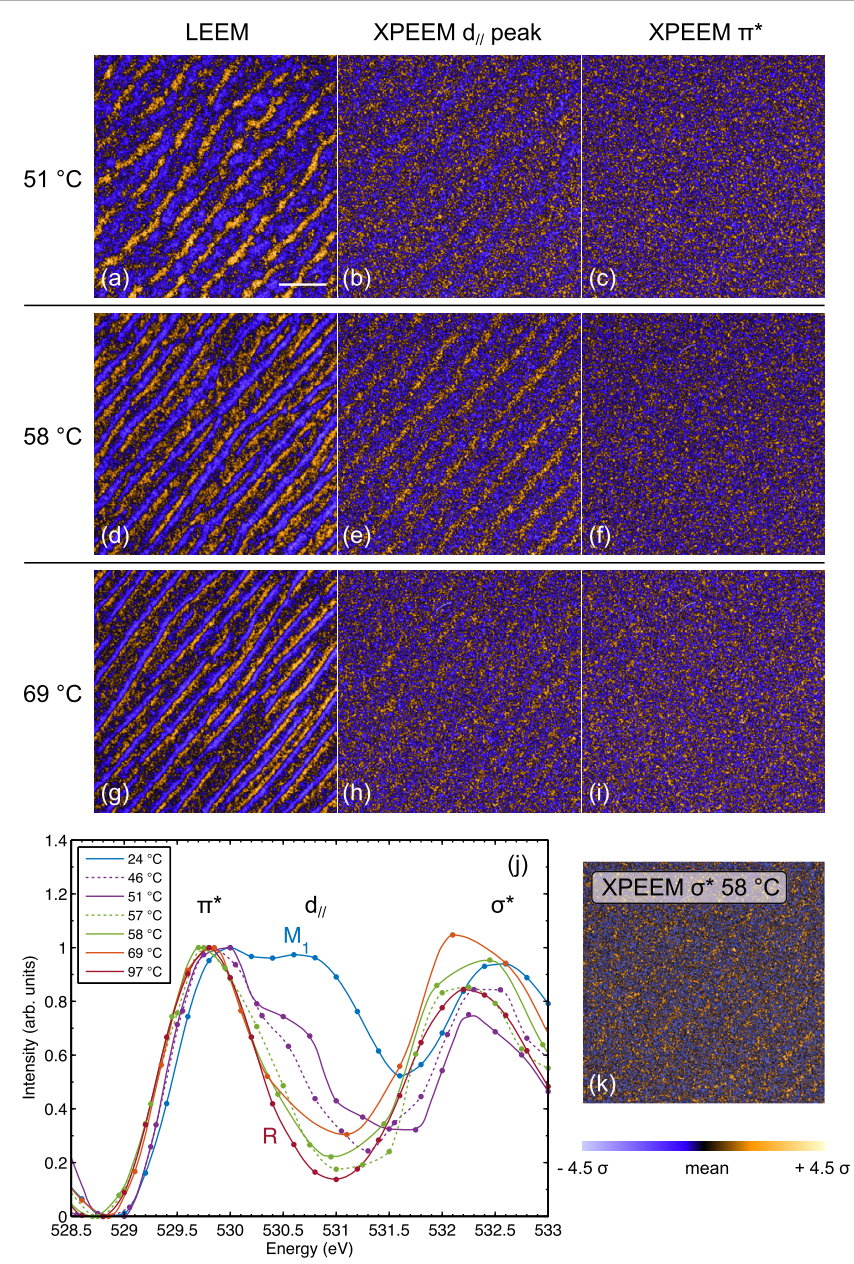

FIG. 1. LEEM and XASPEEM images of $\mathrm{VO}_{2}(110)$ at select temperatures through the MIT. (a)-(c) $51^{\circ} \mathrm{C}$. (d)-(f) $58^{\circ} \mathrm{C}$. (g)(i) $69^{\circ} \mathrm{C}$. At each temperature, three images are shown: LEEM, XPEEM at the $d_{\|}$photon energy (531 eV), and XPEEM at the $\pi^{*}$ photon energy (529.7 eV). (j) Spatially averaged O $K$ edge XAS obtained by integrating the energy-dependent XASPEEM images over their field of view (approximately $100 \mu \mathrm{m}^{2}$ ). (k) Example at $58^{\circ} \mathrm{C}$ of the recovery of XPEEM image contrast at the $\sigma^{*}$ absorption feature $(532.5 \mathrm{eV})$. The color scale of each image is normalized to $\pm 4.5 \sigma$ from the mean LEEM or XPEEM $\left(d_{\|}\right)$ values. Scale bar: $1 \mu \mathrm{m}$.

images recorded at the $d_{\|}$energy shown in Figs. 1(b), 1(e), and 1(h), but it is absent in XASPEEM at the $\pi^{*}$ energy [Figs. 1(c), 1(f), and 1(i)] [31]. This strong contrast gradually weakens before becoming faintly visible again just above the $\sigma^{*}$ states [e.g., at $58^{\circ} \mathrm{C}$ in Fig. 1(k)].

We now quantitatively compare XASPEEM and LEEM to understand the nature of the $\mathrm{V}-\mathrm{V}$ pairing in the monoclinic metallic phase. We extract the XAS spectra corresponding to the two LEEM structures by constructing a mask from the LEEM by thresholding its intensity [25]. The results are summarized in Fig. 2. As an example of the process at $58^{\circ} \mathrm{C}$, Figs. 2(c) and 2(d) show the XASPEEM image in the $d_{\|}$energy window, overlaid with two LEEM 

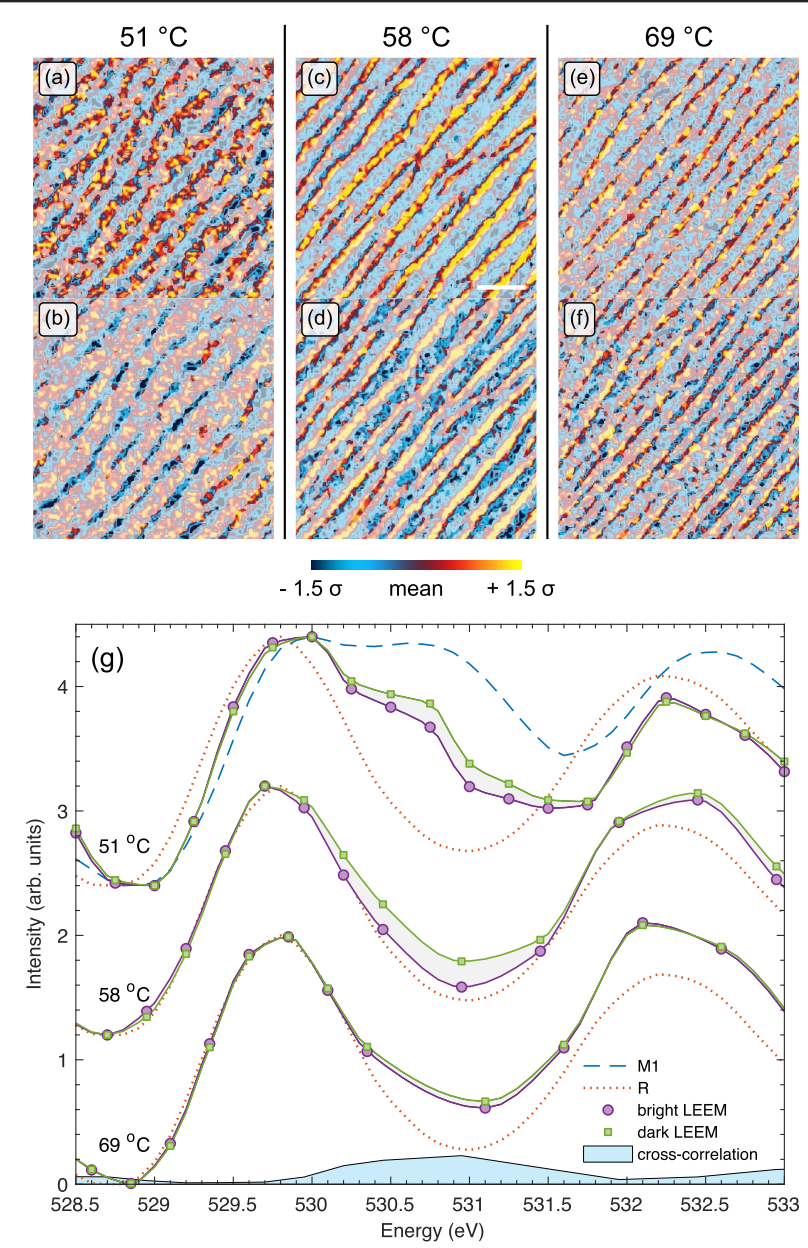

FIG. 2. XASPEEM spectra at select temperatures obtained as a result of applying masks to the energy-dependent XPEEM images. The dark and bright LEEM masks are shown at each temperature in (a)-(f), overlaid on the corresponding $d_{\|}$peak XPEEM images. The thresholds used were $40 \%, 30 \%$, and $30 \%$ for dark masks in (a),(c),(e), and 15\%,33\%, and $40 \%$ for the bright masks in (b),(d),(f). Transparent regions are those that contribute to the XASPEEM spectra. (g) XASPEEM spectra obtained by applying the masks, vertically offset for clarity, and shown alongside reference $M_{1}$ and $R$ phase spectra. Also shown is the rms magnitude of the energy-dependent cross-correlation function at $58^{\circ} \mathrm{C}$. Scale bar: $1 \mu \mathrm{m}$.

masks, corresponding to the dark LEEM intensities [monocliniclike phase, Fig. 2(c)] and bright LEEM intensities [rutilelike phase, Fig. 2(d)]. The agreement between the stripe structure observed in LEEM and XASPEEM at the $d_{\|}$photon energy is excellent, confirming that the contrast in both images has the same spatial origin. Similar results are found at $51{ }^{\circ} \mathrm{C}$ [Figs. 2(a) and 2(b)] and $69^{\circ} \mathrm{C}$ [Figs. 2(e) and 2(f)].

The extracted XASPEEM spectra are shown in Fig. 2(g), alongside the insulating $M_{1}$ and metallic $R$ phase XAS spectra. At $51{ }^{\circ} \mathrm{C}$, there is clear contrast near the $d_{\|}$photon energy, however, the intensity is intermediate between $M_{1}$ and $R$, suggesting that both monocliniclike and rutilelike regions of the sample retain $\mathrm{V}-\mathrm{V}$ dimer bonds to some extent. The "dark LEEM" spectrum, representing monocliniclike $\mathrm{VO}_{2}(110)$, was obtained using a threshold of $40 \%$ of the LEEM pixels at $51{ }^{\circ} \mathrm{C}$, though this spectrum hardly changes at a threshold of $1 \%$, indicating that $M_{1}$ $\mathrm{VO}_{2}$ has already been destroyed. Similarly, the "bright LEEM" spectrum does not approach the $R$ phase spectrum down to a $1 \%$ threshold. In particular, the shapes of the leading edges of the $51{ }^{\circ} \mathrm{C}$ spectra imply that this is a spectrally distinct phase of $\mathrm{VO}_{2}$ [25]. These observations demonstrate that even at this temperature, the intermediate "mixed" phase of strained $\mathrm{VO}_{2}(110)$ contains neither $M_{1}$ nor $R$ phase $\mathrm{VO}_{2}$, and that the contrast in both LEEM and XASPEEM is more complex.

At $58^{\circ} \mathrm{C}$, both extracted spectra show the same leading edge and $\pi^{*}$ structures, supporting the homogeneity of the metallicity. On the other hand, clear contrast between the two spectra is observed at the $d_{\|}$peak, supported by the peak in the rms cross-correlation coefficient [25]. The bright LEEM spectrum approaches the metallic rutile spectrum at all energies, whereas the dark LEEM spectrum exhibits weak enhancement in the absorption coefficient near the $d_{\|}$peak and does not approach the $M_{1}$ phase spectrum at thresholds down to $1 \%$. The monoclinic metallic phase is therefore spectrally distinct from the $M_{1}$ phase, consisting of weakened V-V dimers. Similar results are found at $69^{\circ} \mathrm{C}$, but with reduced contrast and an overall reduction in the intensity at the $d_{\|}$photon energy.

By examining the correlation between the XASPEEM image at the $d_{\|}$energy and the LEEM image, we are able to assess how closely related the spatial variations in crystal (LEEM) and dimerization (XASPEEM) structures are [1,32]; auto- and cross-correlation coefficients at different temperatures are shown in Fig. 3. Perpendicular to the stripe axis, the images [Figs. 3(a)-3(c)] show strong oscillatory behavior typical of stripe order [1], from which we estimate the stripe widths as $235 \pm 12\left(51^{\circ} \mathrm{C}\right), 207 \pm 18\left(58^{\circ} \mathrm{C}\right)$, and $170 \pm 33 \mathrm{~nm}\left(69^{\circ} \mathrm{C}\right)$, measured from the mean first zero crossing (with standard error). Note that the damping of these oscillations along the perpendicular axis is similar in each correlation coefficient, suggesting equivalent long-range order in both LEEM and XASPEEM contrast mechanisms. At $51^{\circ} \mathrm{C}$ [Fig. 3(d)], there is excellent agreement between structures in the LEEM and XASPEEM auto- and crosscorrelations, indicating that the two contrast mechanisms are strongly coupled. However, at $58^{\circ} \mathrm{C}$ [Fig. 3(e)], these begin to diverge and, crucially, are qualitatively different from one another. For example, the second satellites are found at $1.28 \mu \mathrm{m}$ in the LEEM [Fig. 3(a)], compared to $870 \mathrm{~nm}$ in the XASPEEM [Fig. 3(c)] autocorrelations. These are separated from the first satellites (which represent the mean stripe separation, $580 \mathrm{~nm}$ from LEEM and $520 \mathrm{~nm}$ from XASPEEM) by a negative trough, which has a specific meaning here [25]. Similar behavior is observed at $69^{\circ} \mathrm{C}$ [Fig. 3(f)]. This disparity in the structure of the correlation 

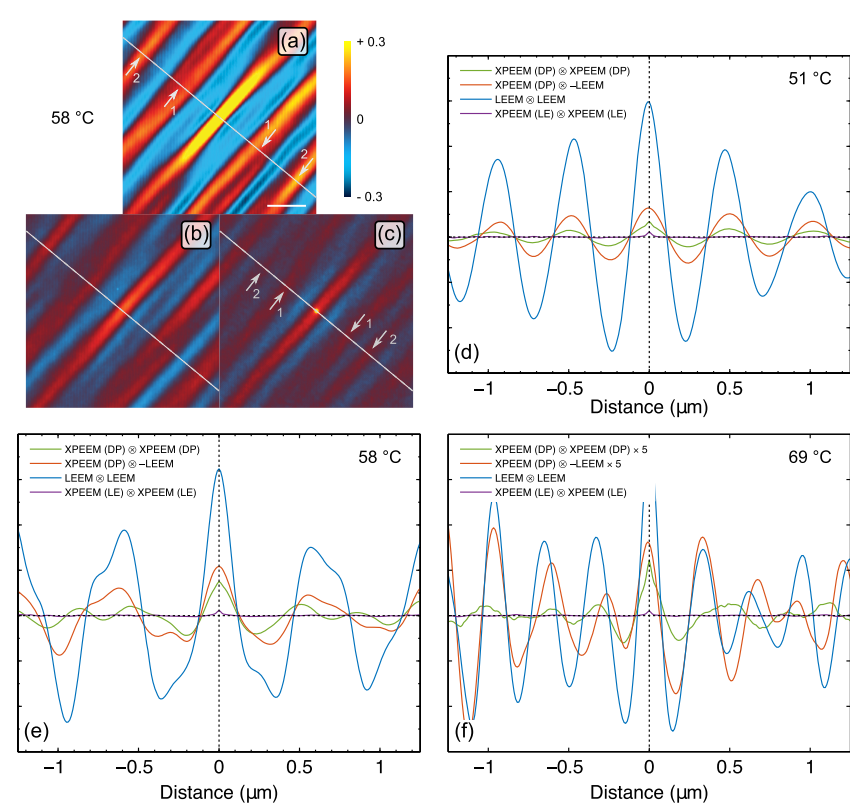

FIG. 3. Auto- and cross-correlation of XASPEEM and LEEM at select temperatures. (a)-(c) Examples of 2D image correlation at $58^{\circ} \mathrm{C}$. (a) Autocorrelation of LEEM. (b) Cross-correlation of (inverse) LEEM and XASPEEM at the $d_{\|}$photon energy (530 to $531 \mathrm{eV}$ ). (c) Autocorrelation of XASPEEM at the $d_{\|}$photon energy. The origin is at the center of each image. Arrows in (a),(c) indicate the first and second satellites. Scale bar: $0.5 \mu \mathrm{m}$. (d),(e), (f) Auto- and cross-correlation profiles at the three temperatures, obtained by integrating along the stripe axis.

above $58^{\circ} \mathrm{C}$ is not expected if the mechanism for contrast in the two measurement techniques is sensitive to the same underlying structure, i.e., in our case, if the crystal symmetry and $\mathrm{V}-\mathrm{V}$ dimerization are directly and causally linked. Nevertheless, it is clear from Fig. 3, and in Figs. 1 and 2 above, that sufficiently similar patterns are observed in both images that the two contrasts are related. We therefore conclude that the symmetry and $\mathrm{V}-\mathrm{V}$ dimerization are different, albeit related, responses to the structural and electronic instabilities that develop in the system in this intermediate part of the phase diagram.

Given the above results, we can establish a detailed picture of the progression of the MIT in strained $\mathrm{VO}_{2}(110)$. Initially, $\mathrm{VO}_{2}$ is insulating and resides in the $M_{1}$ structure, as is well known. With raising temperature, the strict $M_{1}$ structure is quickly lost, as the dimerization of the $\mathrm{V}$ ions along the rutile $c_{R}$ axis is weakened, though the system remains electronically insulating. By $51^{\circ} \mathrm{C}$, the system separates into more strongly and weakly dimerized $\mathrm{V}-\mathrm{V}$ bonds, yielding XASPEEM stripe contrast at the $d_{\|}$energy. Initially, the spatial variation in the magnitudes of the dimer strength and the monoclinic distortion are strongly coupled to one another. Upon further increasing the temperature, the $\mathrm{V}-\mathrm{V}$ bonds are weakened further, resulting in a decrease in intensity of the XASPEEM near the $d_{\|}$photon energy. By $58^{\circ} \mathrm{C}$, the system transitions to a metallic state, but this
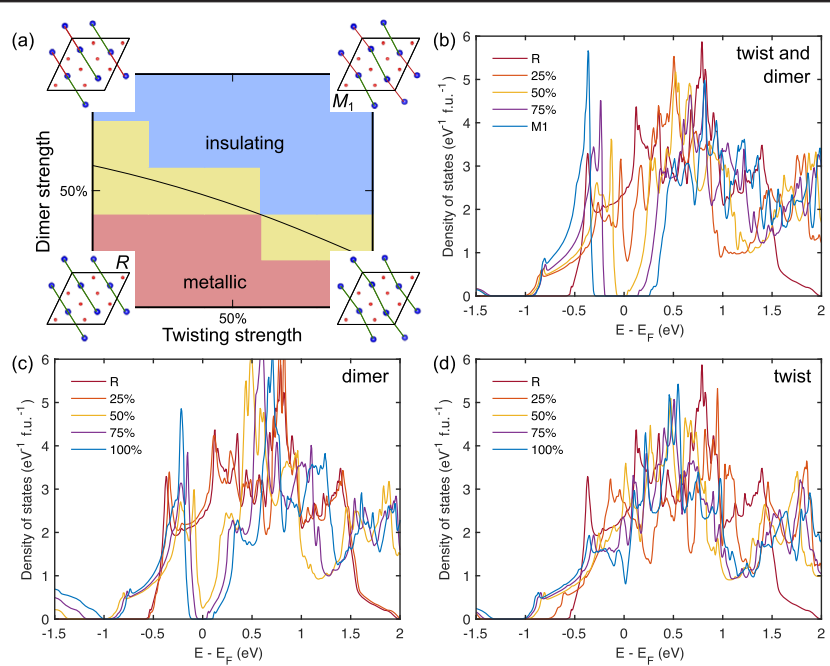

FIG. 4. Total density of states of $\mathrm{VO}_{2}$ from GGA $+U$ calculations as a function of independent evolution in the bond length and monoclinic distortion (twisting of the dimer and $\mathrm{VO}_{6}$ octahedra). (a) Solutions of calculations, with illustrations depicting the nature of the two distortions. (b) Variations in both $\mathrm{V}-\mathrm{V}$ bond length and monoclinic distortion between $R$ and $M_{1}$ phases. (c) Variations in the dimer strength ( $\mathrm{V}-\mathrm{V}$ bond length) only, without monoclinic distortion. (d) Variations in the twisting of the $\mathrm{VO}_{6}$ octahedra (monoclinic distortion) only for constant $\mathrm{V}-\mathrm{V}$ bond length. In each figure, $100 \%$ indicates the $M_{1}$ value.

transition does not follow the underlying stripe structure. Rather, we suggest that once a critical mean $\mathrm{V}-\mathrm{V}$ bond length is reached, the metallic phase is stabilized and propagates rapidly through the sample. Although there exist short-range correlations between the monoclinic distortion and $\mathrm{V}-\mathrm{V}$ dimerization, these are not commensurate with one another over long length scales. Above this temperature, however, monocliniclike stripes remain over a wide temperature range, corresponding to weak dimers in a distorted rutile structure. In terms of the mechanism of the MIT, our key observation is that the metallic phase is not correlated with the stripe structure but rather advances in a narrow temperature range independent of the variations in crystal structure, which is easily reconciled in the presence of strong electron correlations.

It is pertinent to ask what role, if any, other lower symmetry phases of $\mathrm{VO}_{2}$ might play in our interpretation $[8,16]$. It is well known that the monoclinic $M_{2}$ phase, e.g., consists of one half of the $\mathrm{V}-\mathrm{V}$ ions arranged in a twisted (undimerized) 1D chain, and the other half arranged in a dimerized (untwisted) chain (compared to fully dimerized and twisted $\mathrm{V}-\mathrm{V}$ bonds in $M_{1} \mathrm{VO}_{2}$ ) [14]. However, the $M_{2}$ phase is an insulator with a sizable gap, and experimental XAS spectra bear little resemblance to those of our intermediate phase [33]. The triclinic $T$ phase, although insulating with a band gap comparable to the $M_{1}$ and $M_{2}$ phases $[13,18]$, is transitional between these two structures, whereby the equally paired and twisted dimers in $M_{1}$ gradually and continuously differentiate into the structures 
in $M_{2}$ [14]. The $T$ phase illustrates the inherent structural instability of the $\mathrm{V}-\mathrm{V}$ dimers, and that a continuous evolution in their strength and orientation towards a gradual breaking of these bonds is already well established in $\mathrm{VO}_{2}$, which has recently been suggested to play a role in the $M_{1} \rightarrow R$ transition of bulk $\mathrm{VO}_{2}$ [22].

In order to test our interpretations, we have performed electronic structure calculations of $\mathrm{VO}_{2}$ using ELK code [34] within the generalized gradient approximation with a Hubbard term $(\mathrm{GGA}+U)$ formalism $(U=2.8 \mathrm{eV})$ [22], explicitly separating the two structural components of the MIT into independent evolutions in the $\mathrm{V}-\mathrm{V}$ bond length and the twisting of the dimer and $\mathrm{VO}_{6}$ octahedra within the unit cell [25]. All solutions using the rutile $\mathrm{V}-\mathrm{V}$ bond length (i.e., undimerized) are metallic, Figs. 4(a) and 4(d). On the other hand, varying the bond lengths between those of the rutile and $M_{1}$ phases leads to an insulating solution for dimer strengths of $50 \%$ and greater towards the $M_{1}$ structure [Fig. 4(c)]. The significance of this result is that for "weak dimerization," characterized by dimers of $\leq 25 \%$ of the $M_{1}$ bond strengths, GGA $+U$ predicts that $\mathrm{VO}_{2}$ is metallic, irrespective of the twisting of the dimer and $\mathrm{VO}_{6}$ octahedra [Figs. 4(b) and 4(d)]. In the presence of biaxial strain, these intermediate structures may be metastable during the progress of the MIT, in qualitative agreement with our XASPEEM results.

Without strong electron correlations, the band gap in insulating $M_{1} \mathrm{VO}_{2}$ is anticipated to be small $[13,35]$. Partly, this may be explained by the reduced screening available in the $M_{1}$ phase due to the emptying of the $\pi^{*}\left(e_{g}^{\pi}\right)$ orbital and the narrowing of the occupied $d_{\|}\left(a_{1 g}\right)$ due to a reduction in mixing [13]. It is therefore noteworthy that contrast spectra (i.e., the difference between dark LEEM and bright LEEM spectra) based on our XASPEEM results indicate a broader width $(\approx 1.4 \mathrm{eV})$ at $\geq 58^{\circ} \mathrm{C}$ (i.e., in the metallic phases). In the insulating phases $\left(\leq 51{ }^{\circ} \mathrm{C}\right)$, the contrast spectra have a width of $\approx 1.0 \mathrm{eV}$, comparable to the difference spectrum of $\left(M_{1}-R\right)$. Together with the details of the stripes discussed above, these results represent strong evidence that electron correlations are central to the MIT mechanism in $\mathrm{VO}_{2}$.

In summary, the $M_{1} \mathrm{VO}_{2}$ is quickly destroyed on heating and is replaced by an insulating monoclinic phase that gradually separates into strongly and weakly paired dimers that are coupled to the underlying symmetry of the crystal structure. The electronic transition occurs over a narrow temperature range within this structural evolution, giving way to a monocliniclike metallic phase that still contains bimodal dimer pairing and symmetry. The continuous weakening of the structural distortion eventually establishes the rutile phase. These details are inconsistent with a structural MIT model, depending instead on the effects of strong electron correlation.

Data are available at the University of Bristol data repository, data.bris [36].
The Boston University program is supported in part by the Department of Energy under Grant No. DE-FG0298ER45680. S. K., J. W. L., and S. A. W. gratefully acknowledge financial support from the Army Research Office through MURI Grant No. W911-NF-09-1-0398. J. L. acknowledges the EPSRC Centre for Doctoral Training in Condensed Matter Physics (CDT-CMP), Grant No. EP/L015544/1. Calculations were performed using the computational facilities of the Advanced Computing Research Centre, University of Bristol.

[1] J. Laverock, S. Kittiwatanakul, A. A. Zakharov, Y. R. Niu, B. Chen, S. A. Wolf, J. W. Lu, and K. E. Smith, Direct Observation of Decoupled Structural and Electronic Transitions and an Ambient Pressure Monocliniclike Metallic Phase of $\mathrm{VO}_{2}$, Phys. Rev. Lett. 113, 216402 (2014).

[2] E. Arcangeletti, L. Baldassarre, D. Di Castro, S. Lupi, L. Malavasi, C. Marini, A. Perucchi, and P. Postorino, Evidence of a Pressure-Induced Metallization Process in Monoclinic $\mathrm{VO}_{2}$, Phys. Rev. Lett. 98, 196406 (2007); W.-P. Hsieh, M. Trigo, D. A. Reis, G. A. Artioli, L. Malavasi, and W. L. Mao, Evidence for photo-induced monoclinic metallic $\mathrm{VO}_{2}$ under high pressure, Appl. Phys. Lett. 104, 021917 (2014).

[3] B.-J. Kim, Y. W. Lee, S. Choi, J.-W. Lim, S. J. Yun, H.-T. Kim, T.-J. Shin, and H.-S. Yun, Micrometer x-ray diffraction study of $\mathrm{VO}_{2}$ films: Separation between metal-insulator transition and structural phase transition, Phys. Rev. B 77, 235401 (2008); Z. Tao, T.-R. T. Han, S. D. Mahanti, P. M. Duxbury, F. Yuan, C.-Y. Ruan, K. Wang, and J. Wu, Decoupling of Structural and Electronic Phase Transitions in $\mathrm{VO}_{2}$, Phys. Rev. Lett. 109, 166406 (2012); T. L. Cocker, L. V. Titova, S. Fourmaux, G. Holloway, H.-C. Bandulet, D. Brassard, J.-C. Kieffer, M. A. El Khakani, and F. A. Hegmann, Phase diagram of the ultrafast photoinduced insulator-metal transition in vanadium dioxide, Phys. Rev. B 85, 155120 (2012).

[4] F. J. Morin, Oxides which show a Metal-to-Insulator Transition at the Néel Temperature, Phys. Rev. Lett. 3, 34 (1959); N. F. Mott, Metal-Insulator Transitions (Taylor \& Francis, London, 1974).

[5] J. B. Goodenough and H. Y. -P. Hong, Structures and a twoband model for the system $\mathrm{V}_{1-x} \mathrm{Cr}_{x} \mathrm{O}_{2}$, Phys. Rev. B 8, 1323 (1973).

[6] J. Jeong, N. Aetukuri, T. Graf, T. D. Schladt, M. G. Samant, and S. S. P. Parkin, Suppression of metal-insulator transition in $\mathrm{VO}_{2}$ by electric field-induced oxygen vacancy formation, Science 339, 1402 (2013).

[7] A. Cavalleri, C. Töth, C. W. Siders, and J. A. Squier, Femtosecond Structural Dynamics in $\mathrm{VO}_{2}$ During an Ultrafast Solid-Solid Phase Transition, Phys. Rev. Lett. 87, 237401 (2001).

[8] J. H. Park, J. M. Coy, T. S. Kasirga, C. Huang, Z. Fei, S. Hunter, and D. H. Cobden, Measurement of a solid-state triple point at the metal-insulator transition in $\mathrm{VO}_{2}$, Nature (London) 500, 431 (2013).

[9] M. Nakano, K. Shibuya, D. Okuyama, T. Hatano, S. Ono, M. Kawasaki, Y. Iwasa, and Y. Tokura, Collective bulk 
carrier delocalization driven by electrostatic surface charge accumulation, Nature (London) 487, 459 (2012); K. Appavoo, B. Wang, N. F. Brady, M. Seo, J. Nag, R. P. Prasankumar, D. J. Hilton, S. T. Pantelides, and R. F. Haglund, Jr., Ultrafast phase transition via catastrophic phonon collapse driven by plasmonic hot-electron injection, Nano Lett. 14, 1127 (2014); K. Liu, C. Cheng, Z. Cheng, K. Wang, R. Ramesh, and J. Wu, Giant-amplitude, high-work density microactuators with phase transition activated nanolayer bimorphs, Nano Lett. 12, 6302 (2012).

[10] Y. Muraoka and Z. Hiroi, Metal-insulator transition of $\mathrm{VO}_{2}$ thin films grown on $\mathrm{TiO}_{2}(001)$ and (110) substrates, Appl. Phys. Lett. 80, 583 (2002).

[11] J. Cao, E. Ertekin, V. Srinivasan, W. Fan, S. Huang, H. Zheng, J. W. L. Yim, D. R. Khanal, D. F. Ogletree, J. C. Grossman, and J. Wu, Strain engineering and onedimensional organization of metal-insulator domains in single-crystal vanadium dioxide beams, Nat. Nanotechnol. 4, 732 (2009).

[12] M. W. Haverkort, Z. Hu, A. Tanaka, W. Reichelt, S. V. Streltsov, M. A. Korotin, V. I. Anisimov, H. H. Hsieh, H.-J. Lin, C. T. Chen, D. I. Khomskii, and L. H. Tjeng, OrbitalAssisted Metal-Insulator Transition in $\mathrm{VO}_{2}$, Phys. Rev. Lett. 95, 196404 (2005).

[13] A. Zylbersztejn and N. F. Mott, Metal-insulator transition in vanadium dioxide, Phys. Rev. B 11, 4383 (1975).

[14] J. P. Pouget, H. Launois, T. M. Rice, P. Dernier, A. Gossard, G. Villeneuve, and P. Hagenmuller, Dimerization of a linear Heisenberg chain in the insulating phases of $\mathrm{V}_{1-x} \mathrm{Cr}_{x} \mathrm{O}_{2}$, Phys. Rev. B 10, 1801 (1974).

[15] J. P. Pouget, H. Launois, J. P. D’Haenens, P. Merenda, and T. M. Rice, Electron Localization Induced by Uniaxial Stress in Pure $\mathrm{VO}_{2}$, Phys. Rev. Lett. 35, 873 (1975); G. Villeneuve, A. Bordet, A. Casalot, J. P. Pouget, H. Launois, and P. Lederer, Contribution to the study of the metal-insulator transition in the $\mathrm{V}_{1-x} \mathrm{Nb}_{x} \mathrm{O}_{2}$ system: ICrystallographic and transport properties, J. Phys. Chem. Solids 33, 1953 (1972).

[16] Y. Gu, J. Cao, J. Wu, and L.-Q. Chen, Thermodynamics of strained vanadium dioxide single crystals, J. Appl. Phys. 108, 083517 (2010).

[17] J. D. Budai, J. Hong, M. E. Manley, E. D. Specht, C. W. Li, J. Z. Tischler, D. L. Abernathy, A. H. Said, B. M. Leu, L. A. Boatner, R. J. McQueeney, and O. Delaire, Metallization of vanadium dioxide driven by large phonon entropy, Nature (London) 515, 535 (2014).

[18] T. J. Huffman, C. Hendriks, E. J. Walter, J. Yoon, H. Ju, R. Smith, G. L. Carr, H. Krakauer, and M. M. Qazilbash, Insulating phases of vanadium dioxide are Mott-Hubbard insulators, Phys. Rev. B 95, 075125 (2017).

[19] S. Kumar, J. P. Strachan, M. D. Pickett, A. Bratkovsky, Y. Nishi, and R. S. Williams, Sequential electronic and structural transitions in $\mathrm{VO}_{2}$ observed using x-ray absorption spectromicroscopy, Adv. Mater. 26, 7505 (2014).

[20] J. Laverock, A. R. H. Preston, D. Newby Jr., K. E. Smith, S. Sallis, L. F. J. Piper, S. Kittiwatanakul, J. W. Lu, S. A. Wolf, M. Leandersson, and T. Balasubramanian, Photoemission evidence for crossover from Peierls-like to Mott-like transition in highly strained $\mathrm{VO}_{2}$, Phys. Rev. B 86, 195124 (2012).
[21] S. Kittiwatanakul, S. A. Wolf, and J. W. Lu, Large epitaxial bi-axial strain induces a Mott-like phase transition in $\mathrm{VO}_{2}$, Appl. Phys. Lett. 105, 073112 (2014); M. Yang et al., Suppression of structural phase transition in $\mathrm{VO}_{2}$ by epitaxial strain in vicinity of metal-insulator transition, Sci. Rep. 6, 23119 (2016).

[22] T. Yao, X. Zhang, Z. Sun, S. Liu, Y. Huang, Y. Xie, C. Wu, X. Yuan, W. Zhang, Z. Wu, G. Pan, F. Hu, L. Wu, Q. Liu, and S. Wei, Understanding the Nature of the Kinetic Process in a $\mathrm{VO}_{2}$ Metal-Insulator Transition, Phys. Rev. Lett. 105, 226405 (2010).

[23] M. Abbate, F. M. F. de Groot, J. C. Fuggle, Y. J. Ma, C. T. Chen, F. Sette, A. Fujimori, Y. Ueda, and K. Kosuge, Soft-X-ray-absorption studies of the electronic-structure changes through the $\mathrm{VO}_{2}$ phase transition, Phys. Rev. B 43, 7263 (1991); T. C. Koethe, Z. Hu, M. W. Haverkort, C. Schüßler-Langeheine, F. Venturini, N. B. Brookes, O. Tjernberg, W. Reichelt, H. H. Hsieh, H.-J. Lin, C. T. Chen, and L. H. Tjeng, Transfer of Spectral Weight and Symmetry Across the Metal-Insulator Transition in $\mathrm{VO}_{2}$, Phys. Rev. Lett. 97, 116402 (2006).

[24] J. Laverock, L. F. J. Piper, A. R. H. Preston, B. Chen, J. McNulty, K. E. Smith, S. Kittiwatanakul, J. W. Lu, S. A. Wolf, P.-A. Glans, and J.-H. Guo, Strain dependence of bonding and hybridization across the metal-insulator transition of $\mathrm{VO}_{2}$, Phys. Rev. B 85, 081104(R) (2012).

[25] See Supplemental Material at http://link.aps.org/ supplemental/10.1103/PhysRevLett.121.256403 for information on sample growth, details on the experimental techniques and data processing, and information on the electronic structure calculations.

[26] K. G. West, J. Lu, J. Yu, D. Kirkwood, W. Chen, Y. Pei, J. Claassen, and S. A. Wolf, Growth and characterization of vanadium dioxide thin films prepared by reactive-biased target ion beam deposition, J. Vac. Sci. Technol. A 26, 133 (2008).

[27] S. Kittiwatanakul, J. Laverock, D. Newby, Jr., K. E. Smith, S. A. Wolf, and J. Lu, Transport behavior and electronic structure of phase pure $\mathrm{VO}_{2}$ thin films grown on $c$-plane sapphire under different $\mathrm{O}_{2}$ partial pressure, J. Appl. Phys. 114, 053703 (2013).

[28] E. Bauer, Low energy electron microscopy, Rep. Prog. Phys. 57, 895 (1994).

[29] E. Bauer, Photoelectron spectromicroscopy: Present and future, J. Electron Spectrosc. Relat. Phenom. 114, 975 (2001).

[30] J. Laverock, S. Kittiwatanakul, A. A. Zakharov, Y. R. Niu, B. Chen, J. Kuyyalil, S. A. Wolf, J. W. Lu, and K. E. Smith, Simultaneous spectroscopic, diffraction and microscopic study of the metal-insulator transition of $\mathrm{VO}_{2}$, Mater. Res. Soc. Symp. Proc. 1730, 480 (2015).

[31] Note that the contrast in Figs. 1(b), 1(e), and 1(h) is inverted with respect to the LEEM images, indicating that higher absorption at $d_{\|}$is associated with the monocliniclike structural phase observed in LEEM, in agreement with XAS $[23,24]$.

[32] A. S. McLeod, E. van Heumen, J. G. Ramirez, S. Wang, T. Saerbeck, S. Guenon, M. Goldflam, L. Anderegg, P. Kelly, A. Mueller, M. K. Liu, I. K. Schuller, and D. N. Basov, Nanotextured phase coexistence in the correlated insulator $\mathrm{V}_{2} \mathrm{O}_{3}$, Nat. Phys. 13, 80 (2017). 
[33] N.F. Quackenbush et al., Stability of the $M_{2}$ phase of vanadium dioxide induced by coherent epitaxial strain, Phys. Rev. B 94, 085105 (2016).

[34] J. K. Dewhurst, S. Sharma, L. Nordstöm, F. Cricchio, F. Bultmark, and E. K. U. Gross, The ELK FP-LAPW code, http://elk.sourceforge.net (2016).

[35] V. Eyert, The metal-insulator transitions of $\mathrm{VO}_{2}$ : A band theoretical approach, Ann. Phys. (Berlin) 11, 650 (2002); S. Biermann, A. Poteryaev, A. I. Lichtenstein, and A. Georges, Dynamical Singlets and Correlation-Assisted Peierls Transition in $\mathrm{VO}_{2}$, Phys. Rev. Lett. 94, 026404 (2005); B. Lazarovits, K. Kim, K. Haule, and G. Kotliar,
Effects of strain on the electronic structure of $\mathrm{VO}_{2}$, Phys. Rev. B 81, 115117 (2010); V. Eyert $\mathrm{VO}_{2}$ : A Novel View from Band Theory, Phys. Rev. Lett. 107, 016401 (2011); W. H. Brito, M. C. O. Aguiar, K. Haule, and G. Kotliar, Metal-Insulator Transition in $\mathrm{VO}_{2}$ : A DFT + DMFT Perspective, Phys. Rev. Lett. 117, 056402 (2016); O. Nájera, M. Civelli, V. Dobrosavljević, and M. J. Rozenberg, Resolving the $\mathrm{VO}_{2}$ controversy: Mott mechanism dominates the insulator-to-metal transition, Phys. Rev. B 95, 035113 (2017).

[36] J. Laverock, VO2 XAS-PEEM data, University of Bristol, https://doi.org/10.5523/bris.230svfjkbc4b824jiezcf4qe9n, 2018. 